\title{
Spiral Structure Based Limits on the Disk Mass of the Low Surface Brightness Galaxies UGC 6614 and F568-6
}

\author{
A. C. Quillen ${ }^{1,2}$ \& T. E. Pickering ${ }^{1,3}$
}

\begin{abstract}
The spiral structure of the low surface brightness galaxies F568-6 (Malin 2) and UGC 6614 is large scale, with arms that wrap more than half a revolution, and extend out to 50 and $80 \mathrm{kpc}$ in UGC 6614 and F568-6 respectively. The density contrasts observed in the H I maps are high, with arm/interarm contrasts of $\sim 2: 1$, whereas the velocity perturbations due to spiral structure are low, in the range $10-20 \mathrm{~km} / \mathrm{s}$ and 10-30 km/s in UGC 6614 and F568-6 respectively.

Upper limits for the disk mass-to-light ratios are estimated by considering the minimum velocity perturbations in the $\mathrm{H}$ I velocity field that should result from the spiral structure observed in the $R$ band images. The strongest limits occur at small radii ( $r \sim 40^{\prime \prime}$ or $16 \mathrm{kpc}$ and $r \sim 30^{\prime \prime}$ or $24 \mathrm{kpc}$ in UGC 6614 and F568-6 respectively) where there are strong distortions observed in the $R$ band images. The weak observed response in the $\phi$ velocity component limits the mass-to-light ratios of the disk in these regions to $M / L \lesssim 3$ and 6 for UGC 6614 for F568-6 respectively (in solar units) based upon azimuthal variations observed in the $R$ band images. These limits are sufficiently strong to require a significant dark matter component even in the central regions of these galaxies, confirming the findings of previous studies. Our limits furthermore imply that this dark matter component cannot be in the form of a cold disk since a cold disk would necessarily be involved in the spiral structure.

To produce the large observed arm/interarm H I density variations it is likely that the spiral arm potential perturbation is sufficiently strong to produce shocks in the gas. For a forcing that is greater than $2 \%$ of the axisymmetric force, $M / L \gtrsim 1$ is required in both galaxies in the outer regions. This is equivalent to a disk surface density between $r=60-120^{\prime \prime}$ in UGC 6614 of 2.6-1.0 $M_{\odot} / \mathrm{pc}^{2}$ and between $r=40-90^{\prime \prime}$ in F568-6 of 6.6-1.0 $M_{\odot} / \mathrm{pc}^{2}$ assuming that the amplitude of the variations in the disk mass is the same as that observed in the $R$ band. These lower limits imply that the stellar surface density is at least of the same order as the gas surface density. This is consistent with the large scale morphology of the spiral structure, and the stability of the gas disk, both which suggest that a moderate stellar component is required to produce the observed spiral structure.
\end{abstract}

\footnotetext{
${ }^{1}$ University of Arizona, Steward Observatory, Tucson, AZ 85721

${ }^{2}$ E-mail: aquillen@as.arizona.edu

${ }^{3}$ E-mail: tim@as.arizona.edu
} 
Subject headings: galaxies: structure — galaxies: spiral — galaxies: low surface brightness galaxies

\section{Introduction}

The observed rotation curve shapes of both giant, so-called Malin-type, low surface brightness galaxies (e.g. de Blok \& McGaugh 1996, Pickering et al. 1997) and dwarf low surface brightness galaxies (e.g. de Blok \& McGaugh 1996, van Zee et al. 1997) do not match the rotation curves shapes inferred from their light distributions assuming constant mass-to-light ratios. In both cases, the observed rotation curves rise more slowly and continue rising out to radii where the rotation curves inferred from the light distributions are declining. In the giant low surface brightness galaxies, very high disk mass-to-light ratios of about 20-30 (in $R$ band) would be required to match the high observed rotation speeds (Pickering et al. 1997). As a result, to fit the rotation curves of these galaxies a substantial dark matter component is required, even at small radii, and they are said to be dark matter dominated. This is in contrast to normal high surface brightness galaxies where maximal disk solutions with lower mass-to-light ratios yield good fits to the rotation curves (e.g. Kent 1987a \& Kent 1987b) in the central few disk scale lengths. Comparison of low and high surface brightness galaxies with equal total luminosity suggest that low surface brightness galaxies have low mass surface density disks (de Blok et al. 1996). This is consistent with the fact that these galaxies appear at near normal locations on the Tully Fisher relation (Zwaan et al. 1995).

In spite of their low mass surface densities, some low surface brightness disk galaxies do show spiral structure including, of course, the two cases discussed here as well as all of the giant low surface brightness galaxies presented in Sprayberry et al. (1995) and some of the dwarfs in the samples of van Zee et al. 1997 and de Blok et al. 1996 (e.g. UGC 11820, UGC 5716, and F568-1). Since spiral structure requires non-axisymmetric mass perturbations in the disk of the galaxy itself, its properties can be used to limit the mass of the disk involved in the spiral density waves. If velocity perturbations caused by the spiral structure are small, a limit can be placed on the total mass in the spiral structure, yielding an upper limit on the mass-to-light ratio of the luminous stellar disk. Low surface brightness galaxies can show spiral structure despite the fact that their gas densities fall below the critical stability limit (Kennicutt 1989) for instability due to spiral structure (van der Hulst et al. 1993, de Blok et al. 1996). This suggests that a stellar disk component is required so that the disk is sufficiently unstable to produce the observed spiral arms (Pickering et al. 1997). If evidence for strong spiral shocks is seen in the gas response, then a sufficiently strong spiral gravitational force is required to produce this gas response. This critical forcing yields a lower limit for the mass of the disk.

Indeed the concept of using spiral arm patterns to limit the disk mass-to-light ratio

was considered by Visser 1980 who compared the predictions of spiral density wave theory 
to observations of M81. He stated "The second test is whether the amplitude of the wave as measured" (by photometry) "converted into the amplitude of the spiral arm potential perturbation, is consistent with the observed amplitude of the density and velocity perturbations of the gas." He treated the forcing spiral wave amplitude as a free parameter and found that large amplitudes produced velocity residuals too large to be consistent with the observations, whereas weak forcing amplitudes did not produce shocks leading to the large density contrasts which are observed in H I emission in M81. Using a different approach, Athanassoula et al. 1987 placed limits on the mass-to-light ratios of some normal disk galaxies by assuming that the disks should amplify $m=2$ or bisymmetric spiral modes but inhibit asymmetric $m=1$ modes.

Low surface brightness galaxies are an important setting to place limits on the mass in the disk for two reasons: 1) They have low density disks and 2) They appear to be dark matter dominated. Normal high surface brightness galaxies with strong spiral structure have bright disks which are adequately massive to produce a strong gravitational spiral force even when the spiral structure is relatively weak. However, in the low surface brightness galaxies the disk surface brightness is 1-3 magnitudes fainter even though rotational velocities are nearly equivalent so it is quite surprising that spiral structure exists in any of these galaxies. Many normal galaxies can be well fit by maximal disk or near maximal disk rotation curve models (e.g. Kent 1987a, Kent 1987b) and models of spiral gas response require gravitational forcing which is consistent with the amplitude variations observed from images of these disks (e.g. Lowe et al. 1994). By placing limits on the mass density of the disk from the existence of spiral structure we can test the degree to which these galaxies are dark matter dominated and the possibility that these galaxies might have a massive disk component.

In this paper we use the spiral structure observed in optical and H I images of the low surface brightness galaxies UGC 6614 and F568-6 (Malin 2) to place both upper and lower limits on the mass-to-light ratio of the optical disk. In $\S 2$ we review the $\mathrm{H}$ I and $R$ band images which show spiral structure in these two galaxies. In $\S 3$ we place upper limits on the mass-to-light ratio of this spiral structure based on the low level of spiral arm induced velocity perturbations. In $\S 4$ we consider the mass density in spiral structure required to produce shocks in the gas that would be consistent with the large density contrasts observed in the $\mathrm{H}$ I emission maps. A discussion follows in $\S 5$.

\section{Spiral Structure in UGC 6614 and F568-6}

UGC 6614 and F568-6 were observed in $R$ band and in H I by Pickering et al. 1997 to investigate their neutral hydrogen and kinematic properties. These data are displayed as overlays in Figures 1 and 2 and are described in detail in Pickering et al. 1997. UGC 6614 and F568-6 are both low surface brightness galaxies with central surface brightnesses several magnitudes fainter than sky level $\left(\mu_{R}(0)=22.9\right.$ and 22.1 for UGC 6614 and F568-6 respectively). Their disk exponential scale lengths are large (14 and 18 kpc for UGC 6614 and F568-6 respectively) and 
they both contain copious amounts of H I $\left(2.5\right.$ and $3.6 \times 10^{10} M_{\odot}$ for the two galaxies respectively, Pickering et al. 1997) We assume here the distances of $D=85$ and $164 \mathrm{Mpc}$ to UGC 6614 and F568-6 respectively (following Pickering et al. 1997; derived from a Hubble constant of $75 \mathrm{Mpc}$ $\mathrm{km} / \mathrm{s})$.

Both galaxies show clear evidence of spiral structure (see Figures 1 and 2). Spiral arms are visible in the optical images, the $\mathrm{H}$ I emission maps, and as kinks in the velocity field at the level of $10-30 \mathrm{~km} / \mathrm{s}$. This spiral arm structure is coincident in the $R$ band and $\mathrm{H}$ I images as well as in the velocity field. The spiral structure for the two galaxies is large scale extending to a radius of more than 50 and $90 \mathrm{kpc}$ in UGC 6614 and F568-6 respectively. This is in contrast to normal galaxies such as M81 where the entire spiral arm structure lies within the optical disk or within $\sim 20 \mathrm{kpc}$.

\subsection{Spiral Arm Morphology}

For both galaxies the morphology of the spiral structure consists of coherent spiral arms each of which wrap around the galaxy more than half a revolution. In this respect the spiral structure does not resemble that of flocculent late-type galaxies where pieces of arms exist only locally. Both spiral structures are strongly asymmetric, particularly in their inner regions. The outer arms in UGC 6614, however, are close to being a bi-symmetric or two arm spiral pattern which is centered about a position located to the east of the nucleus. In flocculent galaxies, the spiral structure is thought to be primarily propagating in the gas disk without strong coupling to the stellar disk (e.g. Braine et al. 1993). Also, simulations have shown that when the disk mass is low, the spiral structure is more likely to be flocculent (Sellwood \& Carlberg 1984, Carlberg \& Freedman 1985). The more coherent nature of the spiral structure observed in these low surface brightness galaxies suggests that the stellar disk is coupled to the gas disk and actively involved in the spiral structure. The gas disks of these galaxies are stable to spiral density waves (McGaugh 1992, van der Hulst et al. 1993, Pickering et al. 1997), suggesting that a gas plus stellar disk is required for the disk to be sufficiently unstable to support the observed spiral structure.

The H I surface brightness map shows large arm/interarm contrasts of $\sim 2: 1$ and velocity variations seen as kinks in the velocity field detectable at the level of $10-20$ and $10-30 \mathrm{~km} / \mathrm{s}$ in UGC 6614 and F568-6 respectively. We note that these values for the arm/interarm contrast and velocity residuals are similar to those observed in M81 which has radial velocity perturbations of $\sim 10 \mathrm{~km} / \mathrm{s}$ observable in H I (Visser 1980). High surface brightness galaxies can also have significantly stronger velocity perturbations. For example, M51 has radial velocity perturbations of $60-90 \mathrm{~km} / \mathrm{s}$ seen in the CO velocity field (Vogel et al. 1988), and UGC 2885 has perturbations of 50-70 km/s observed in $\mathrm{H} \alpha$ (Canzian et al. 1993).

Along the kinematic minor axis of the galaxy velocity residuals are expected to be radial. The pattern of the velocity residual pattern depends on whether the spiral arm pattern lies within 
the corotation radius (Canzian 1993). Kinks in the velocity field alternate sign only a few times as a function of radius along the minor axes of the two galaxies. This residual pattern which is consistent with a single residual alternating sign pair associated with each arm is similar to the velocity residual field of M81 and suggests that the entire spiral pattern lies within the corotation resonance (Canzian 1993).

\subsection{Spiral Arm Amplitudes}

To estimate the amplitude of the spiral arm structure observed in $\mathrm{H} \mathrm{I}$ and in the $R$ band images, we must first correct for the inclination of the galaxy. This is relatively straightforward in the case of UGC 6614 since the $R$ band isophote, $\mathrm{H}$ I isophote and H I velocity field derived position and inclination angles all agree $\left(\mathrm{PA}=116^{\circ}, i=35^{\circ} \pm 3^{\circ}\right.$, Pickering et al. 1997). For F568-6 the $R$ band isophote and $\mathrm{H}$ I velocity field derived position and inclination angles agree $\left(\mathrm{PA}=75^{\circ}, i=38^{\circ} \pm 3^{\circ}\right)$, though the $\mathrm{H} \mathrm{I}$ distribution is more asymmetric than the $R$ band light (Pickering et al. 1997). We have adopted these orientations to correct for the galaxy inclinations.

In Figures 3 and 4 we show azimuthal profiles in the inclination corrected $R$ band images and $\mathrm{H}$ I maps for the two galaxies. The amplitudes as a function of radius of the $m=1$ and $m=2$ azimuthal Fourier components expressed as a percentage of the azimuthal average for the two galaxies are shown in Figure 5 for the $R$ band images and in Figure 6 for the $\mathrm{H}$ I images. UGC 6614 has an oval distortion in its central region $\left(r<40^{\prime \prime}\right)$ which is particularly noticeable after correction for inclination. (By oval distortion we mean an elongation in the isophotes that does not vary in position angle over a range of radius.) This oval distortion is seen as a large azimuthal density variation (evident as high $m=2$ components in Figure 5) which is not an actual intensity variation in the spiral arms. In the outer regions of both galaxies the faint spiral arms are far brighter than the underlying disk which is only marginally detected. Although peaks in the H I and $R$ band surface brightness are correlated (see Figures 1 and 2) they do not correspond to maximum densities or surface brightnesses in the azimuthal cuts except at large radii in UGC 6614 (see Figures 3 and 4). Better correlation might be observed with higher angular resolution H I observations.

Since the amplitudes shown in Figures 3 and 4 depend on the assumed galaxy orientations we recomputed them for moderate variations in the inclination angles. For a $5^{\circ}$ lower inclination of $30^{\circ}$ in UGC 6614 , the change in amplitudes were largest in the region of the oval distortion $\left(r<40^{\prime \prime}\right)$ and were $10-15 \%$ smaller than at an inclination of $35^{\circ}$. Variations in the amplitudes elsewhere in UGC 6614 and in F568-6 for a corresponding difference in inclination were smaller, $\lesssim 5 \%$. These amplitude uncertaintites are not sufficiently large to significantly change the limits for the mass-to-light ratio we estimate below. 


\section{Placing an Upper Limit on the Mass-to-Light Ratio}

Spiral density waves are a resonant wave phenomenon. Because resonances can exist a small gravitational perturbation can give a large response, however the opposite is not true. Given a particular spiral gravitational perturbation there is a minimum possible response. This makes it possible to place an upper limit upon the strength of the gravitational perturbation if a measure of the response, such as kinks in the velocity field are limited. To place an upper limit on the mass-to-light ratio of the spiral structure, we consider that the mass involved in the spiral density wave is insufficient to drive a strong response. We therefore estimate the smallest possible gas response to a non-axisymmetric or spiral gravitational perturbation. For such a perturbation, an expansion to first order should give an appropriate description for the gas flow in regions not directly affected by resonances. In this section we follow the notation and derivation given in chapter 6 of Binney \& Tremaine 1987,

We assume that there is a non-axisymmetric perturbation to the gravitational potential in the plane of the galaxy of the form

$$
\Phi_{1}(r, \phi)=R e\left[\Phi_{a}(r) \exp i(m \phi-\omega t)\right]
$$

where the pattern speed of the perturbation is $\Omega_{p} \equiv \omega / \mathrm{m}$. We consider perturbations in the gas velocity field in response to this perturbation. The response in velocity to a tightly wound spiral density wave to first order for the $\phi$ (non-radial) component is of the same form as that of the potential and in the tight winding or WKB approximation is (Binney \& Tremaine 1987, equation 6-37)

$$
v_{\phi a}(r)=\frac{-2 B i k\left(\Phi_{a}+h_{a}\right)}{\kappa^{2}-(m \Omega-\omega)^{2}}
$$

where $\kappa$ is the epicyclic frequency, $\Omega$ and $v_{c}=\Omega / r$ are the angular rotation rate and circular velocity of the unperturbed system at radius $r$, and $B \equiv-\kappa^{2} / 4 \Omega$. The wavenumber of the spiral arm perturbation, $k$, is given by $k \equiv d f(r) / d r$ where $\Phi_{a}(r) \propto e^{i f(r)} . h_{a}$ corresponds to variations in the specific enthalpy of the same form as (equation 1) and can be neglected in the limit $c_{s}<<v_{c}$ which is appropriate for the rotation curves of the low surface brightness galaxies considered here.

Although these velocity perturbations become large near resonances, a limit on the minimum possible velocity perturbation can be placed from the observations. We can rewrite the above equation as

$$
v_{\phi a}=\frac{i k \Phi_{a}}{\left(1-(m \Omega-\omega)^{2} \kappa^{-2}\right) 2 \Omega} .
$$

Because the magnitude of the denominator of the above equation reaches a maximum near corotation we can place the approximate limit

$$
\left|v_{\phi a}\right| \gtrsim\left|\frac{k \Phi_{a}}{2 \Omega}\right|
$$


A density perturbation of the same form as Eq. 1 causes the spiral potential perturbation given above where

$$
\Phi_{a}=\frac{-2 \pi G \Sigma_{a}}{|k|}
$$

(Binney \& Tremaine 1987, equation 6-17) for an infinitely thin disk. Subbing this into the above equation gives us the limit

$$
\left|v_{\phi a}\right| \gtrsim\left|\frac{\pi G \Sigma_{a}}{\Omega}\right| .
$$

If we consider the possibility that the stellar component is more massive than the gas component the above expression can be inverted to yield an upper limit on the mass-to-light ratio, $M / L$, of the disk given an upper limit on the $\phi$ component velocity perturbations across the spiral arm:

$$
M / L \lesssim \frac{v_{\phi a} v_{c}}{\pi G S_{a} r}
$$

where $S_{a}$ is the surface brightness variation about radius $r$ (of the same form as Eq. 1), and $v_{\phi a}$ is the maximum velocity perturbation detected (or detectable in the case of no detection).

The $\phi$ component velocity perturbations are particularly noticeable along the kinematic major axis where the line of sight component of the velocity is in the azimuthal direction. From Figures 1 and 2 we can see that for UGC 6614 these velocity perturbations are smaller than $\sim 15 \mathrm{~km} / \mathrm{s}$, whereas for F568-6 larger perturbations are detected but are $\lesssim 25 \mathrm{~km} / \mathrm{s}$. Using these velocities as upper limits and the $m=1$ and $m=2$ components of the surface brightness variations from the azimuthal profiles (shown in Figure 5) upper limits to the mass-to-light ratios as a function of radius for the two galaxies are shown in Figure 7.

The $m=2$ component oval distortion for $r \sim 40^{\prime \prime}$ in UGC 6614 is sufficiently strong to produce larger velocity perturbations than detected if the mass-to-light ratio is greater than $\sim 3$ (for $R$ band in solar units) in this region. From this we derive a limit $M / L \lesssim 3$ in the central region of the galaxy. Alternatively we can think of this limit in terms of mass and say that if the disk has perturbations of the same amplitude as observed in the $R$ band image, the surface density of the disk must be less than $30 M_{\odot} / \mathrm{pc}^{2}$ (for $M / L=3$ and a surface brightness of 23.5 $\mathrm{mag} / \operatorname{arcsec}^{2}$ in the $R$ band at $r=40^{\prime \prime}$ or $16 \mathrm{kpc}$ ). For F568-6 the spiral structure in the central regions is not as strong and only gives a limit of $M / L \lesssim 6$ at $r \sim 30^{\prime \prime}$ which is equivalent to a surface density which must be less than $60 M_{\odot} / \mathrm{pc}^{2}$ (for $M / L=6$ and a surface brightness of 23.5 $\mathrm{mag} / \operatorname{arcsec}^{2}$ in the $R$ band at $r=30^{\prime \prime}$ or $\left.24 \mathrm{kpc}\right)$.

The fits to the rotation curves of Pickering et al. 1997 for both galaxies have $M / L \lesssim 1$ for the bulge and disk and require a substantial dark matter component even at small radii. For these fits the peak of the velocity contribution from the disk is only $\sim 40$ and $90 \mathrm{~km} / \mathrm{s}$ for UGC 6614 and F568-6 respectively. For our upper limit of $M / L=3$ and 6 for the disks in UGC 6614 and F568-6 a substantial dark matter component is still required to reach the observed maximum rotational velocity. Our upper limits reinforce the findings of Pickering et al. 1997 and others based on fits to the rotation curve that a substantial dark matter component is required even in 
the central regions of these low surface brightness galaxies. This limit further requires that the dark component cannot be in the form of a cold stellar disk (which would have to be involved in the spiral structure).

The above high stellar surface densities are consistent with our neglect of the gas density in Eq. 7 since they are significantly higher than the gas density (see Figure 5). However, even though strong density variations variations exist in the $\mathrm{H}$ I for these galaxies we have estimated the minimum possible velocity response using linear perturbation theory. We note here that it is possible that the non-linear response of the gas could cause the velocity perturbations to be somewhat smaller than inferred from the above limit. In this case a higher mass-to-light ratio could be consistent with the small size of the observed velocity perturbations. If future higher angular resolution H I observations reveal larger velocity perturbations along the spiral arms, then the upper limit for the mass-to-light ratio would also be higher than stated here. An underlying more massive disk at these radii could exist if it had smaller azimuthal density variations than observed in the $R$ band. We also note that our lowest value for the mass-to-light ratio in UGC 6614 coincided with density perturbations that were part of an oval distortion in the galaxy with position angle that varied only slowly with radius. While it is not unreasonable that this oval distortion could be driving a strong gas response, the WKB or tight winding approximation is no longer valid. Although the minimum velocity response is of the same order as given in Eq. 6, this equation could be inaccurate by a factor of a few.

\section{Critical Forcing Required to Produce Shocks - A Lower Limit for $M / L$}

The large density variations in the spiral structure of UGC 6614 and F568-6 are evidence for shocks in the ISM induced by a spiral gravitational potential. Indeed the high arm/interarm density contrast of H I observed in galaxies such as M81 and M51 is one of the major predictions of the spiral density wave theory. In this section we consider how much mass is required in the form of spiral structure to drive shocks in the gas that would be consistent with the $\sim 2: 1 \mathrm{arm} /$ interarm density contrasts observed in the $\mathrm{H}$ I of these two low surface brightness galaxies.

The response of the gas in a spiral density wave is primarily dependent on the forcing gravitational field and the effective ISM sound speed and only weakly dependent on the cloud dependent properties such as the cloud mean-free path and the cloud number density Roberts \& Hausman 1984, Hausman \& Roberts 1984, and Roberts 1969). A critical forcing parameter to produce shocks or large density contrasts in the ISM was explored by Roberts 1969 and Shu et al. 1973. These authors considered the role of $F$, the spiral gravitational force expressed as percentage of the axisymmetric force. Originally Roberts 1969 found that a forcing of $F>2 \%$ was required to produce a density contrast of greater than 2 and that for $F<1 \%$ no shock was produced. Subsequent work by Shu et al. 1973 found that the critical forcing parameter depended on the effective sound speed of the gas, $c_{g}$, and on the speed of the imposed spiral force $c \equiv m \Omega_{p} / k$. For a wide variety of pattern speeds, Shu et al. 1973 found that a forcing amplitude of at least 
$3-4 \%$ was required to produce shocks in the gas, although when $c / c_{g}$ is moderately greater than 1 , a forcing of only $1 \%$ could produce shocks. This differed from the critical forcing parameter introduced in Toomre 1977 (see also Binney \& Tremaine 1987), valid in the limit of low effective sound speed, which requires forcing amplitudes of a few percent for moderate values of $c / c_{g}>1$ (Toomre 1977).

Subsequent models and simulations of the gas response find that forcing of at least a few percent is required to produce the observed gas density contrasts. For example using a forcing spiral gravitational field based on photometry of M81 Visser 1980 found that when $F \lesssim 4 \%$ no shocks were produced in his model. Although more recent modeling of M81 does not vary the forcing amplitude, Lowe et al. 1994 produce the observed density contrast with simulations driven by a spiral forcing amplitude of $5-10 \%$. By exploring the properties of cloud-particle simulations Roberts \& Hausman 1984 found that density contrasts of a few resulted where $F=5-10 \%$ using a spiral structure based on M81 even for the low effective gas sound speed of $8 \mathrm{~km} / \mathrm{s}$.

To summarize, the critical forcing value of a few percent ( $\gtrsim 3-4 \%$ ) seems to be required to produce shocks in the ISM that result in significant gas density contrasts, although this value is dependent on $c_{g}$ and $c$ so that an ISM with a low effective sound speed forced by a fast spiral pattern could produce shocks with the weak forcing amplitude of only $\sim 1 \%$.

\subsection{Forcing Amplitudes in F568-6 and UGC 6614}

For the potential given in Eq. 1, the forcing amplitude expressed as a ratio of the unperturbed axisymmetric gravitational force can be written

$$
F \equiv \frac{\Phi_{a} k}{r \Omega^{2}}
$$

Using Eq. 5 we can rewrite this in terms of the density variation as

$$
F=\frac{2 \pi G \Sigma_{a} r}{v_{c}^{2}}
$$

Using the above expression we have computed the forcing amplitudes using $\Sigma_{a}$ estimated from the $m=1$ and $m=2$ gas and $R$ band azimuthal components with $M / L=1$ in the $R$ band. These forcing amplitudes are shown in Figure 8. We note that the forcing amplitudes are typically quite small. The distortions in the central regions of both galaxies are of sufficient strength to drive shocks in the gas assuming a mass-to-light ratio of 1 . In these regions the gas contribution to the spiral gravitational field are negligible. At larger radii the gas and stellar spiral gravitational forcing in both galaxies are quite small, $\lesssim 2 \%$ of the axisymmetric force.

If we assume that a minimum particular forcing strength is required to produce the observed $\mathrm{H}$ I density contrasts, then we derive a lower limit for the mass-to-light ratio of the disk. We have chosen a critical value of $2 \%$ because even though a forcing of only $1 \%$ might be able to cause 
shocks, is unlikely to cause the observed gas density contrast. Larger values typical of normal galaxies such as M81 might be inconsistent with the low rate of star formation observed in these galaxies (Pickering et al. 1997). Assuming that forcing of at least $2 \%$ is required to produce the observed H I density contrasts then in both galaxies we derive the lower limit $M / L \gtrsim 1$ in the outer parts of the disks $\left(r>60^{\prime \prime}\right.$ or $24 \mathrm{kpc}$ and $r>40^{\prime \prime}$ or $32 \mathrm{kpc}$ in UGC 6614 and F568-6 respectively). This can be expressed as a mass density (if the underlying stellar disk has the same amplitude azimuthal variations as observed in $R$ band). In UGC 6614 for $r=60-120^{\prime \prime}$ the $R$ band surface brightness varies from $25-26 \mathrm{mag} / \operatorname{arcsec}^{2}$ which for $M / L=1$ is equivalent to a disk stellar surface density of 2.6-1.0 $M_{\odot} / \mathrm{pc}^{2}$. In F568-6 for $r=40-90^{\prime \prime}$ the $R$ band surface brightness varies from $24-26 \mathrm{mag} / \operatorname{arcsec}^{2}$ which for $M / L=1$ is equivalent to a disk stellar surface density of 6.6-1.0 $M_{\odot} / \mathrm{pc}^{2}$. These lower limits imply that the stellar surface density is of the same order as the gas surface density. This is consistent with the large scale morphology of the spiral structure and the stability of the gas disk, both of which suggest that a moderate stellar component is required to produce the observed spiral structure.

We note that the forcing assuming $M / L=1$ given in Figure 8 (expressed as a ratio of the unperturbed axisymmetric gravitational force) is smaller than derived for normal high surface brightness galaxies but not 10 times smaller as expected from the 1-3 lower magnitude surface brightness disks of the low surface brightness galaxies, and the equivalent size of their rotational velocites. This is due to the fact that at the large radii where spiral structure exists in these low surface brightness galaxies, the axisymmetric force $\left(v_{c}^{2} / r\right)$ is lower than that at the smaller radii where the spiral structure exists in normal galaxies.

One possible concern is that a moderate external tidal field could be driving the gas response instead of a spiral disk component. In this case the disks of these galaxies could have very low mass-to-light ratios. However, UGC 6614 appears to be isolated; no gas near its redshift was evident in the velocity channel maps of Pickering et al. 1997. On the other hand, in F568-6 there is evidence for interacting material; there is a high velocity clump of gas to the south of the nucleus which seems to be due to a superimposed, possibly interacting dwarf (Pickering et al. 1997). This scenario is unlikely, though, because an external tidal field should cause an oval (non-spiral) perturbation to the gravitational potential which would drive spiral shocks in the gas that are more open than the tightly wound spiral arms observed. It is also difficult for an external tidal field to drive shocks in the gas over a large range of radius. An external tidal field could however be ultimately responsible for exciting the spiral density waves in the gas plus stellar disk (as is likely in the case of M51), but then the resulting stellar and gas derived spiral gravitational field must be sufficiently strong to cause shocks in the gas as assumed here.

\section{Summary and Discussion}

The spiral structure of the low surface brightness galaxies F568-6 and UGC 6614 is large scale, with arms that wrap more than half a revolution, and extend out to 50 and $80 \mathrm{kpc}$ in UGC 
6614 and F568-6 respectively. These spiral arms are visible in the $R$ band images, the $\mathrm{H}$ I emission maps and as kinks in the $\mathrm{H}$ I velocity fields. The density contrasts observed in the H I maps are high, with arm/interarm contrasts of $\sim 2: 1$, whereas the velocity perturbations due to spiral structure are low, in the range $10-20 \mathrm{~km} / \mathrm{s}$ and $10-30 \mathrm{~km} / \mathrm{s}$ in UGC 6614 and F568-6 respectively.

We use the small velocity response to place upper limits on the mass-to-light ratio of the stellar disk. The strongest limits occur at small radii $\left(r \sim 40^{\prime \prime}\right.$ or $16 \mathrm{kpc}$ and $r \sim 30^{\prime \prime}$ or $24 \mathrm{kpc}$ in UGC 6614 and F568-6 respectively) where there are strong distortions observed in the $R$ band images. The weak observed response in the $\phi$ velocity component limits the mass-to-light ratios of the disk in these regions to $M / L<3$ and 6 for UGC 6614 for F568-6 respectively. This is equivalent to requiring the densities of the disks (if they have azimuthal variations of the same size as that observed in the $R$ band images) to be less than 30 and $60 M_{\odot} / \mathrm{pc}^{2}$ at a radius of 16 and $24 \mathrm{kpc}$ for UGC 6614 and F568-6 respectively. An underlying more massive disk at these radii could exist if it had smaller azimuthal density variations than observed in the $R$ band. These limits are sufficiently strong to require a significant dark matter component even in the central regions of this galaxy, confirming the findings of previous studies. Our limits furthermore imply that this dark matter component cannot be in the form of a cold disk since a cold disk would necessarily be involved in the spiral structure, though a hot disk cannot be excluded. We note that this upper limit was derived assuming a linear gas response in the tight winding or WKB approximation, and that a non-linear gas response driven by bar like or oval distortions could cause small velocity perturbations to be present even in the case of stronger potential perturbations (or higher mass-to-light ratios).

To produce the large arm/interarm H I density variations it is likely that the spiral arm potential perturbation is sufficiently strong to produce shocks in the gas. For a forcing that is greater than $2 \%$ of the axisymmetric force, $M / L \gtrsim 1$ is required in both galaxies in the outer regions. This is equivalent to a disk surface density between $r=60-120^{\prime \prime}$ in UGC 6614 of $2.6-1.0$ $M_{\odot} / \mathrm{pc}^{2}$ and between $r=40-90^{\prime \prime}$ in F568-6 of 6.6-1.0 $M_{\odot} / \mathrm{pc}^{2}$ assuming that the amplitude of the variations in the disk mass is the same as that observed in the $R$ band. These lower limits imply that the stellar surface density is of the same order as the gas surface density. This is consistent with the large scale morphology of the spiral structure, and the stability of the gas disk, both of which suggest that a moderate stellar component is required to produce the observed spiral structure. The gas disks alone are not unstable to spiral density waves (Pickering et al. 1997), however it is likely that the combined stellar and gas disks are (see Jog \& Solomon 1984 for instability in a two fluid disk). The coupled gas and stellar disk would be consistent with the large scale spiral arm morphologies which do not resemble that of gas dominated flocculent galaxies.

The limits for the mass-to-light ratio of the disk derived here suggest that the disks of these two low surface brightness galaxies lie in the range $1<M / L<6$ (in the $R$ band). This range is identical to that found for normal higher surface brightness disk galaxies derived from maximal disk fits to the observed rotation curves (Kent 1987a, Kent 1987b). In other words the disk mass-to-light ratio limits placed here are not abnormal compared to those of normal galaxies. Our 
limits on the disk surface densities remain consistent with previous studies which find that low surface brightness galaxies have substantially lower mass surface densities than normal galaxies (de Blok et al. 1996, Sprayberry et al. (1995b)).

We note that multi-wavelength observations (particularly those in the infrared) find that amplitude variations across spiral arms can be a strong function of wavelength (e. g. Rix \& Rieke 1993). While the low H I column depths in UGC 6614 and F568-6 suggest that extinction from dust is not a large effect in these low surface brightness galaxies, it would not be surprising if an older population of stars (which would be more apparent in the near infrared wavelengths) might have smaller spiral arm amplitudes. In this case a lower limit for the mass-to-light derived as we have done here but from a near infrared image might yield even stronger limits requiring an even more massive stellar disk. If high mass-to-light ratios are required, then a mass-to-light ratio variation across the disk could be required to yield a good fit to the rotation curve assuming a smooth halo component, and a large dark matter component might not necessarily be required in the central regions. Multi-wavelength observations coupled with metalicity measurements should also provide useful information about what kinds of stellar populations would be consistent with the range of mass-to-light ratios given here.

It might prove to be interesting to place similar mass-to-light ratio limits in low surface brightness dwarf galaxies which also can have spiral structure (for example UGC 11820, UGC 5716, and F568-1, from van Zee et al. 1997 and de Blok et al. 1996). These galaxies can have regular, symmetrical H I velocity fields and smooth H I emission maps, even when the optical components lack symmetry or contain strong spiral structure. The optical components therefore don't strongly influence the velocity field (although some of these effects might be visible in H I observations at higher angular resolution). This suggests that the upper limits discussed here might be particularly revealing.

We acknowledge helpful discussions and correspondence with R. Kennicutt, L. van Zee, G. Rieke, J. Navarro and H-W. Rix. 


\section{REFERENCES}

Athanassoula, E., Bosma, A., \& Papaioannou, S. 1987, A\&A, 179, 23

Binney, J., \& Tremaine, S. 1987, Galactic Dynamics, (Princeton U. Press).

Braine, J., Combes, F., \& van Driel, W. 1993, A\&A, 280, 451

Canzian, B., Allen, R. J., \& Tilanus, R. P. J. 1993, ApJ, 406, 457

Canzian, B. 1993, ApJ, 414, 487

Carlberg, R. G., \& Freedman, W. L. 1985, ApJ, 298, 486

de Blok, W. J. G., \& McGaugh, S. S. 1996, ApJ, 469, 89L

de Blok, W. J. G., McGaugh, S. S., \& van der Hulst, J. M. 1996, MNRAS, 283, 18

Elmegreen, B. G., Seiden, P. E., \& Elmegreen, D. M. 1989, ApJ, 343, 602

Hausman, M. A., \& Roberts, W. W., Jr. 1984, ApJ, 282, 106

Jog, C. H., \& Solomon, P. M. 1984, ApJ, 276, 114

Kennicutt, R. C. 1989, ApJ, 344, 685

Kent, S. M. 1987a, AJ, 91, 1301

Kent, S. M. 1987b, AJ, 93, 816

Lowe, S. A., Roberts, W. W., Yang, J., Bertin, G., \& Lin, C. C. 1994, ApJ, 427, 184

McGaugh, S. S. 1992, University of Michigan, Ph. D. Thesis

McGaugh, S. S., Schombert, J. M., \& Bothun, G. D. 1995, AJ, 109, 2019

Pickering, T. E., Impey, C. D., van Gorkom, J. H., \& Bothun, G. D. 1997, submitted to ApJ

Rix, H.-W., \& Rieke M. J. 1993, ApJ, 418, 123

Roberts, W. W., Jr., \& Hausman, M. A. 1984, ApJ, 277, 744

Roberts, W. W., Jr., \& Stewart, G. R. 1987, ApJ, 314, 10

Roberts, W. W., Jr. 1969, ApJ, 158, 123

Sellwood, J. A., \& Carlberg, R. G. 1984, ApJ, 282, 61

Shu, F. H., Milione, V. \& Roberts, W. W. 1973, ApJ, 183, 819

Sprayberry, D., Impey, C. D., Bothun, G. D., \& Irwin, M. J. 1995, AJ, 109, 558 
Sprayberry, D., Bernstein, G. M., Impey, C. D., \& Bothun, G. D. 1995, ApJ, 438, 72

Toomre, A. 1977, ARA\&A, 15, 437

van der Hulst, J. M., Skillman, E. D., Smith, T. R., Bothun, G. D., McGaugh, S. S., \& deBlok, W. J. G. 1993, AJ, 106, 548

van Zee, L., Haynes, M. P., Salzer, J. J., \& Broeils, A. H., 1997, preprint

Visser, H. C. D. 1980, A\&A, 88, 149

Vogel, S. N., Kulkarni, S. R., \& Scoville, N. A. 1988, Nature, 334, 402

Zwaan, M. A., van der Hulst, J. M., de Blok, W. J. G., \& McGaugh, S. S. 1995, MNRAS, 273, $35 \mathrm{~L}$

This preprint was prepared with the AAS $\mathrm{LAT}_{\mathrm{E}} \mathrm{X}$ macros v4.0. 
This figure "fig1.jpg" is available in "jpg" format from: http://arxiv.org/ps/astro-ph/9701159v1 
This figure "fig2.jpg" is available in "jpg" format from: http://arxiv.org/ps/astro-ph/9701159v1 

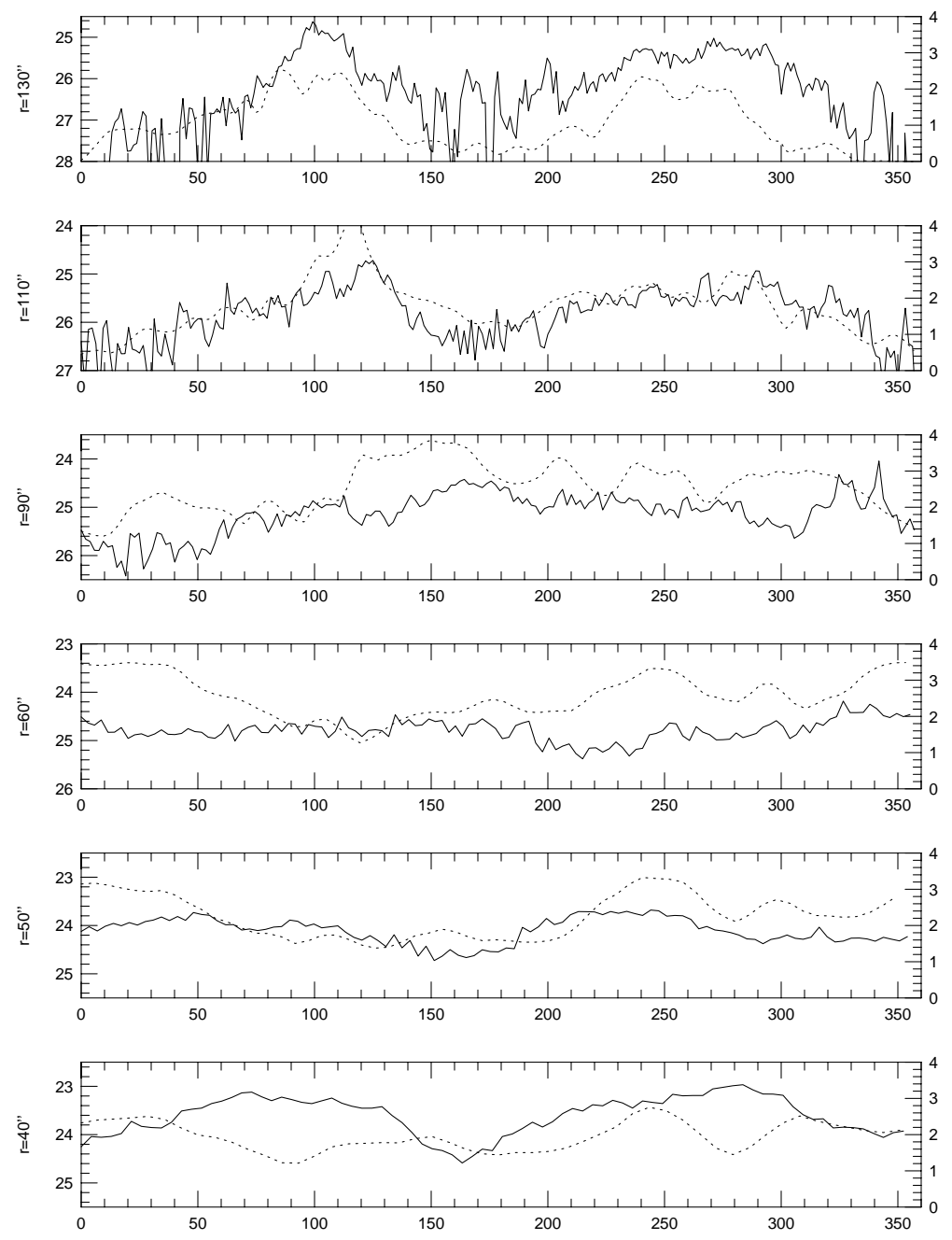

Fig. 3.- Azimuthal profiles as a function of azimuthal angle of the deprojected UGC $6614 R$ band image (solid line) given in mag/ $\operatorname{arcsec}^{2}$ (left vertical axes) and the deprojected H I surface density image (dotted line) in $M_{\odot} / \mathrm{pc}^{2}$ (right vertical axes). On the horizontal axis, $0^{\circ}$ is along the major axis of the galaxy $\left(\mathrm{PA}=287^{\circ}\right)$, and $90^{\circ}$ is perpendicular to this axis in the plane of the galaxy which lies along $\mathrm{PA}=17^{\circ}$. The radius of each azimuthal cut is given on the left hand side of the plot. For small radii $\left(35^{\prime \prime}<r<55^{\prime \prime}\right)$ there is an oval distortion causing the large density variations seen in the azimuthal profiles within these radii. At larger radii, brighter regions lie exclusively along the faint outer spiral arms. The north-eastern spiral arm is brighter than the south-western one. Although peaks in the $\mathrm{H} \mathrm{I}$ and $R$ band surface brightness are correlated (see Figure 1) they do not correspond to maximum densities or surface brightnesses in the azimuthal cuts except at large radii. Better correlation might be observed in higher resolution $\mathrm{H} \mathrm{I}$ data. At larger radii, $\mathrm{H} \mathrm{I}$ dense regions lie exclusively along the outer spiral arms. 

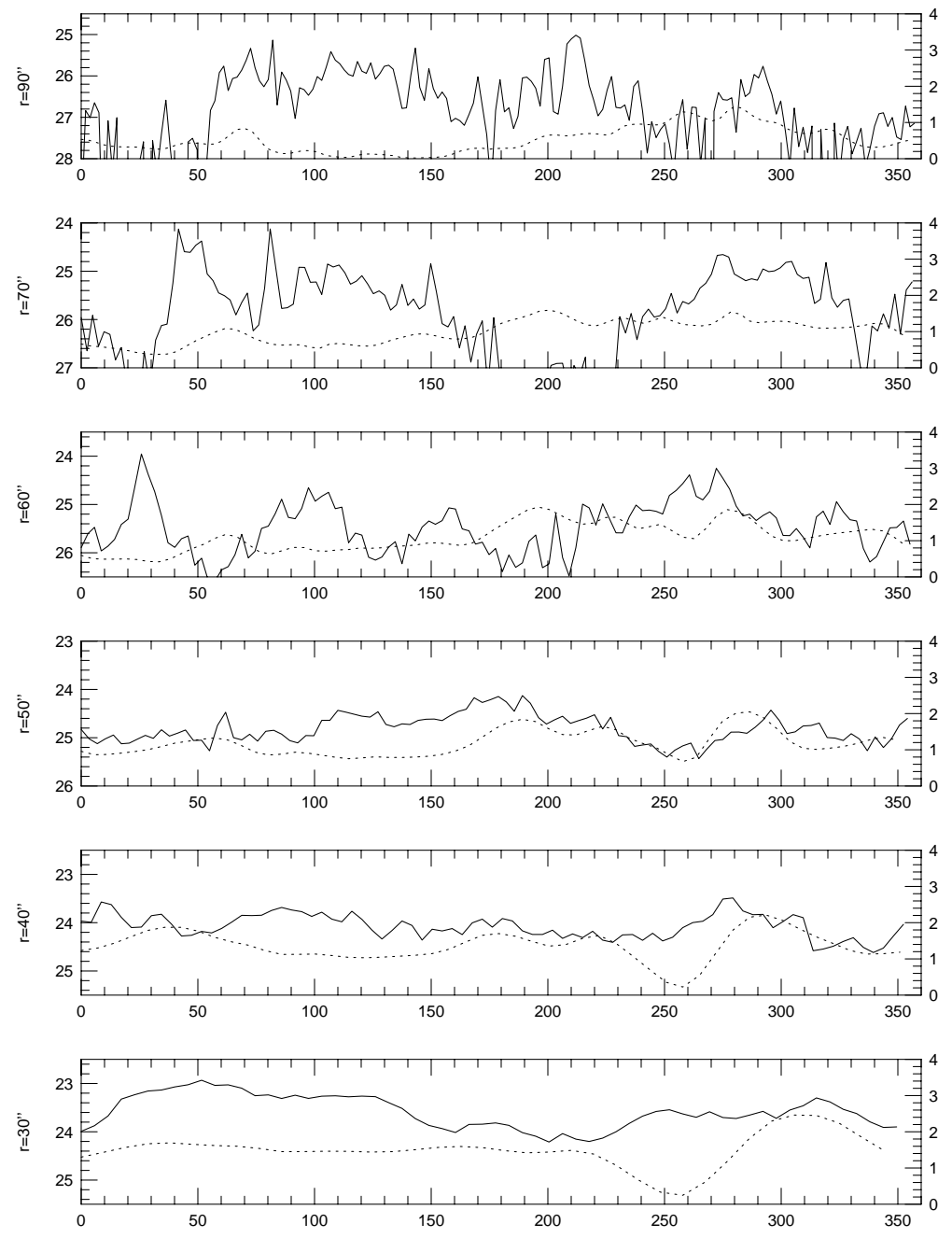

Fig. 4.- Azimuthal profiles as a function of azimuthal angle of the deprojected F568-6 $R$ band image (solid line) given in mag/ $\operatorname{arcsec}^{2}$ (left vertical axes) and the H I surface density image (dotted line) in $M_{\odot} / \mathrm{pc}^{2}$ (right vertical axes). On the horizontal axis, $0^{\circ}$ is along the major axis of the galaxy $\left(\mathrm{PA}=-105^{\circ}\right)$, and $90^{\circ}$ is perpendicular to this axis in the plane of the galaxy which lies along $\mathrm{PA}=-15^{\circ}$. The radius of each azimuthal cut is given on the left hand side of the plot. For small radii $\left(20^{\prime \prime}<r<45^{\prime \prime}\right)$ there is an oval distortion causing the large density variations seen in the azimuthal profiles within these radii. Although peaks in the $\mathrm{H} \mathrm{I}$ and $R$ band surface brightness are correlated (see Figure 2) they do not correspond to maximum densities or surface brightnesses in the azimuthal cuts. Better correlation might be observed in higher resolution H I data. 

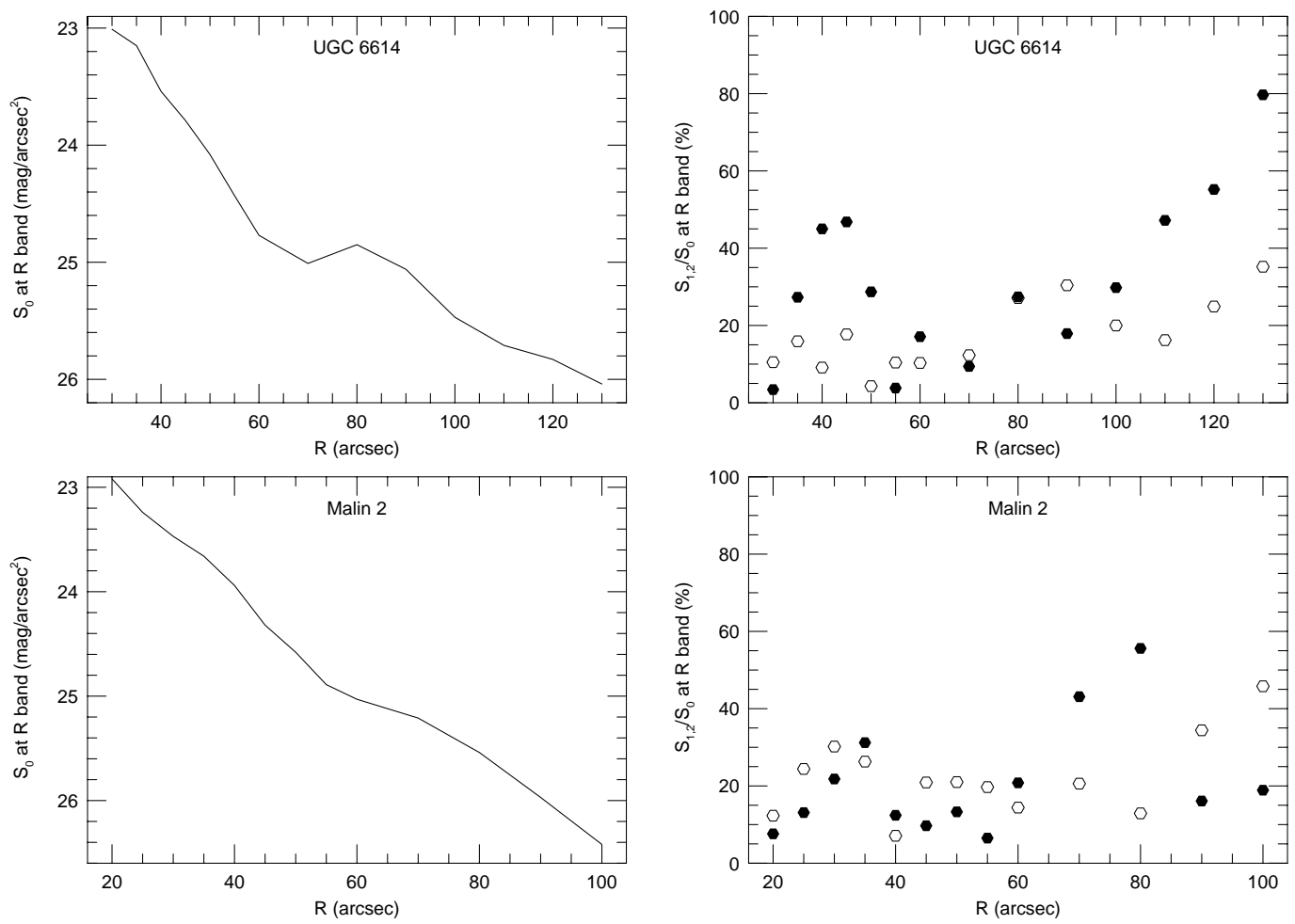

Fig. 5.- a) Azimuthally averaged, inclination corrected $R$ band surface brightness profile for UGC 6614.

b) The $m=2$ (solid points) and $m=1$ (open points) component azimuthal variations in $R$ band for UGC 6614 expressed as a percentage of the azimuthally average value. There is a strong oval distortion at $r \sim 40^{\prime \prime}$. Bi-symmetric or two arm spiral structure is apparent as strong $m=2$ components at large radii in UGC 6614.

c) Azimuthally averaged, inclination corrected $R$ band surface brightness profile for F568-6.

d) The $m=2$ (solid points) and $m=1$ (open points) component azimuthal variations in $R$ band for F568-6 expressed as a percentage of the azimuthally average value. There is a moderate distortion at $r \sim 30^{\prime \prime}$. 

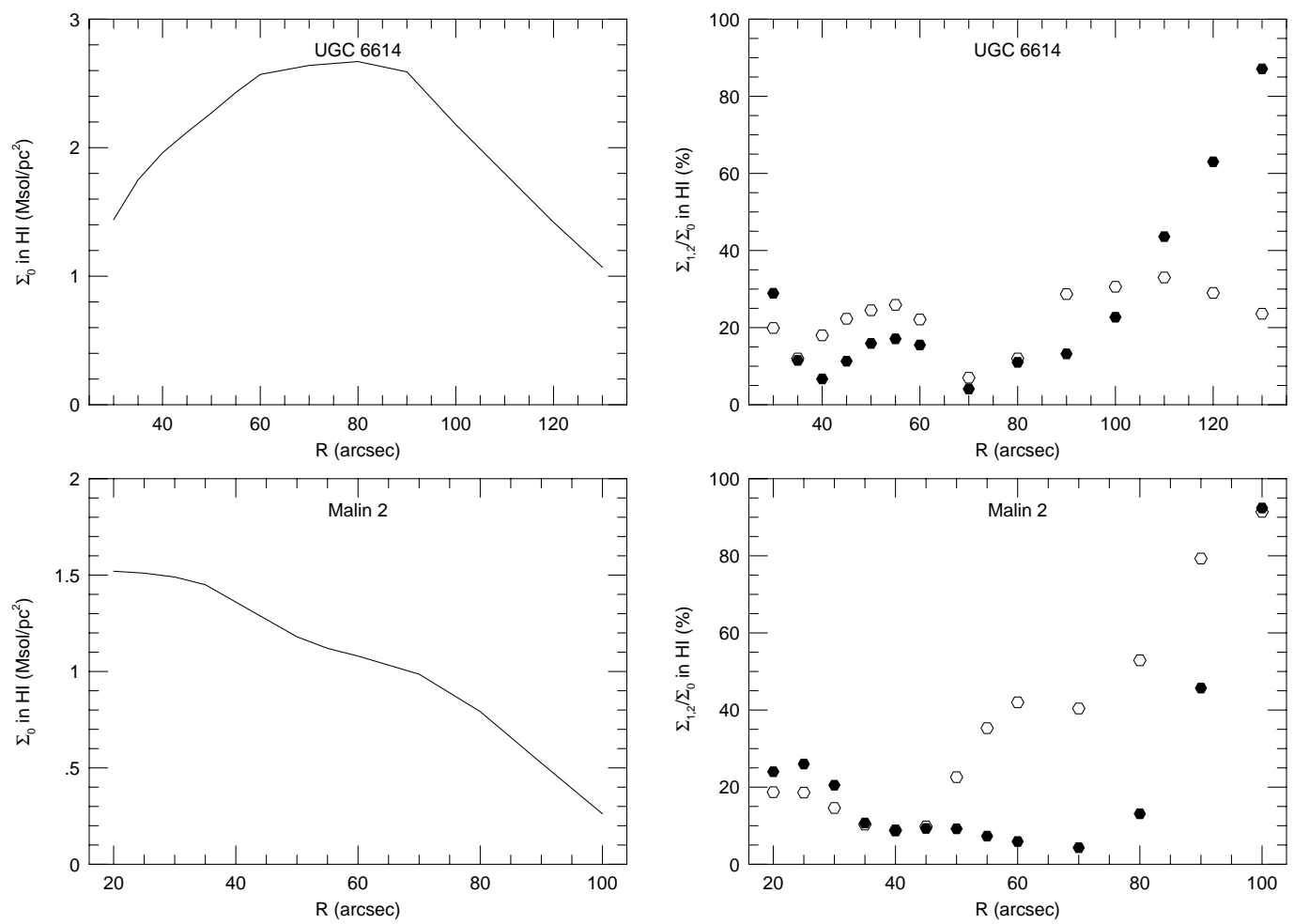

Fig. 6.- a) Azimuthally averaged, inclination corrected gas density profile derived from the H I intensity map of UGC 6614 .

b) The $m=2$ (solid points) and $m=1$ (open points) component azimuthal variations in the gas for UGC 6614 expressed as a percentage of the azimuthally average value.

c) Azimuthally averaged, inclination corrected gas density profile derived from the H I intensity map of F568-6.

d) The $m=2$ (solid points) and $m=1$ (open points) component azimuthal variations in the gas for F568-6 expressed as a percentage of the azimuthally average value. Contrasts are higher at larger radii in both galaxies. 

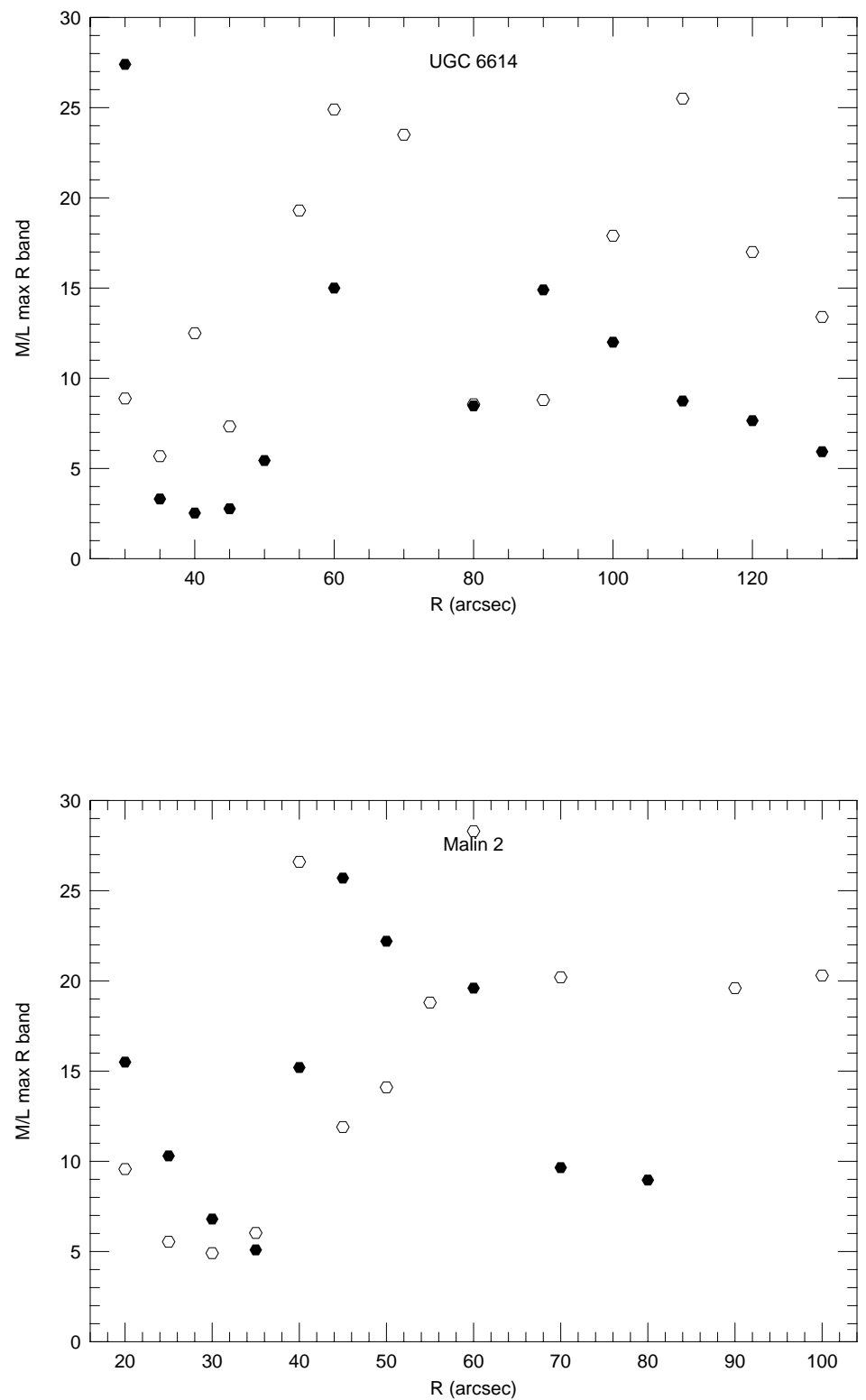

Fig. 7.- a) Upper limits for the mass-to-light ratio, $M / L$, (in solar units) in UGC 6614 based upon the $R$ band $m=2$ (solid points) and $m=1$ (open points) component azimuthal variations assuming that tangential velocity perturbations are no greater than $15 \mathrm{~km} / \mathrm{s}$.

b) Upper limits for the mass-to-light ratio, $M / L$, (in solar units) in F568-6 based upon the $R$ band $m=2$ (solid points) and $m=1$ (open points) component azimuthal variations assuming that tangential velocity perturbations are no greater than $25 \mathrm{~km} / \mathrm{s}$. 

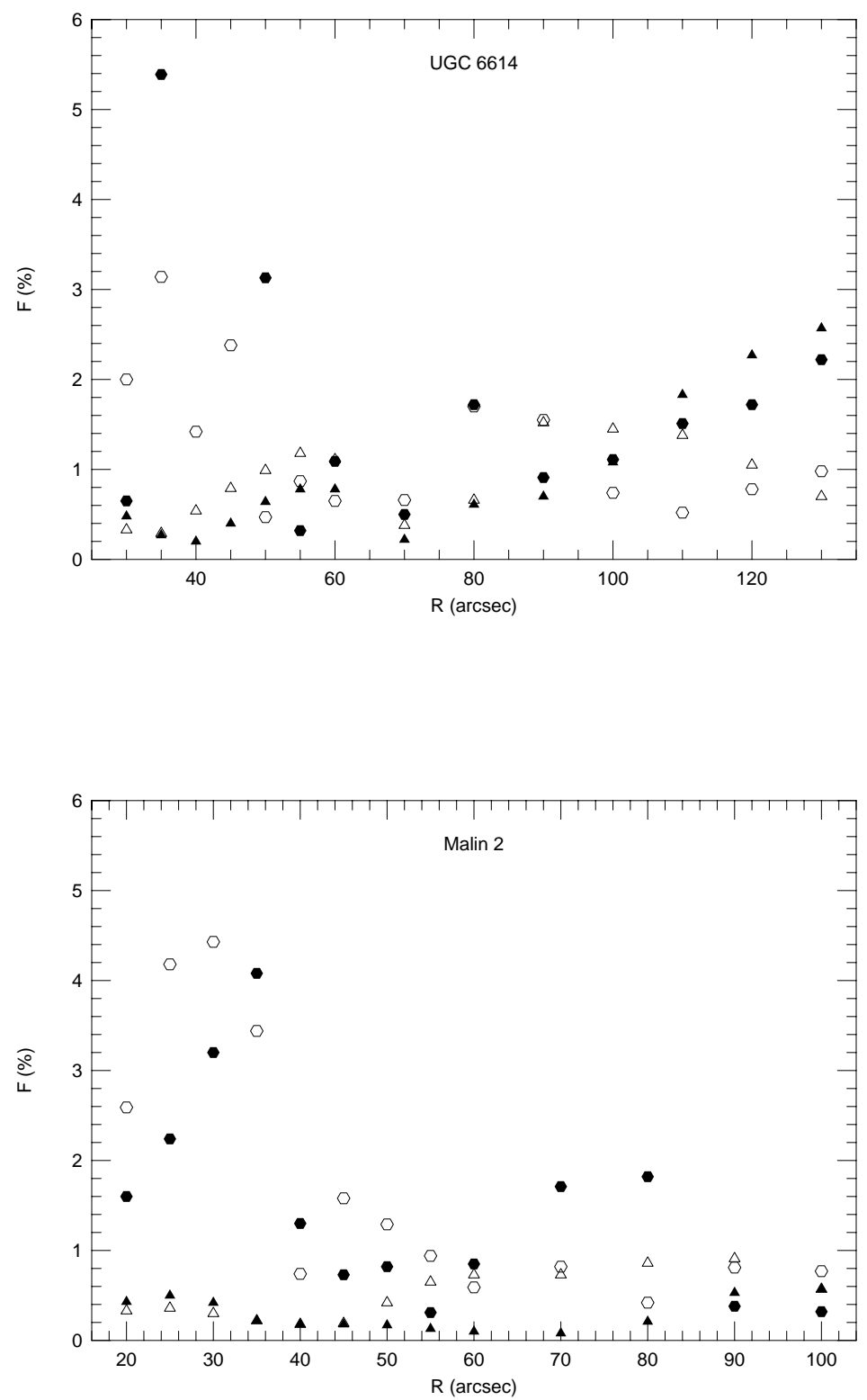

Fig. 8.- a) Spiral forcing in UGC 6614 expressed as a percentage of the axisymmetric force caused by the $R$ band surface brightness variation (hexagons) and the H I surface density (triangles) for the $m=2$ (solid points) and $m=1$ (open points) component variations assuming a mass-to-light ratio of $M / L=1$ in solar units in $R$ band. Note that the spiral forcing is typically quite small. b) Same as a) but in F568-6. 